\title{
Hypotension due to Chemotherapy in a Patient with Small Cell Lung Cancer and Lambert-Eaton Myasthenic Syndrome Undergoing Hemodialysis: A First Case Report
}

\author{
Taiji Kuwata Hidetaka Uramoto Tetsuro Baba \\ Fumihiro Tanaka
}

Second Department of Surgery, School of Medicine, University of Occupational and Environmental Health, Kitakyushu, Japan

\section{Key Words}

Small cell lung cancer · Chemotherapy · Hemodialysis - Lambert-Eaton myasthenic syndrome

\begin{abstract}
We present the first case of small cell lung cancer with Lambert-Eaton myasthenic syndrome during hemodialysis (HD). A 72-year-old male patient receiving HD experienced progressive muscle weakness. He was diagnosed with small cell lung cancer with Lambert-Eaton myasthenic syndrome due to an increased serum level of anti-voltage-gated calcium channel antibody and aspiration cytology on endobronchial ultrasonography for the swelling of a subcarinal lymph node. He received chemotherapy consisting of carboplatin $\left(300 \mathrm{mg} / \mathrm{m}^{2}\right)$ and etoposide $\left(50 \mathrm{mg} / \mathrm{m}^{2}\right)$, to which he had a partial response. However, the second therapy course could not be administered because of the unexpected development of severe hematological adverse events, which also prevented him from undergoing further HD. This case indicates that caution should be taken when using chemotherapy for such patients because of hypotension due to chemotherapy, with which it is impossible to undergo HD.
\end{abstract}

\section{Introduction}

Lambert-Eaton myasthenic syndrome (LEMS) is known to be closely associated with small cell lung cancer (SCLC) in 50-60\% of patients [1], and 2-3\% of SCLC patients also have LEMS [2]. It was reported that the symptoms of LEMS, such as muscle weakness, could be improved by chemotherapy for SCLC $[3,4]$. The agent 3,4-diaminopyridine and intravenous immunoglobulin have been demonstrated to be effective for LEMS [5, 
6]. Lung cancer patients sometimes present with chronic renal failure (CRF). The incidence of lung cancer is thought to be similar in HD patients and non-HD patients [7]. There has recently been an increase in the number of patients on HD. However, the treatment for SCLC patients with LEMS on HD has not been well investigated.

Here, we report the case of a patient who had SCLC with LEMS and who was receiving HD. A chemotherapy regimen consisting of carboplatin (CBDCA) and etoposide (VP-16) was effective; however, HD could not be performed due to the development of severe hypotension. We herein discuss the clinical problems associated with this case and emphasize that physicians should be cautious when treating such patients.

\section{Case Report}

A 72-year-old male with CRF undergoing HD developed lower extremity muscle weakness. He had been examined in the neurology department, and magnetic resonance imaging (MRI) indicated that both his brain and spine were normal. Finally, he was diagnosed as having LEMS because his serum level of anti-voltage-gated calcium channel antibody was extremely elevated (more than 23,000 fmol/l) and electrical stimulation showed waxing of muscle. Chest computed tomography (CT) showed that the right hilar and mediastinal lymph nodes were enlarged. At first, the patient's muscle weakness was treated with 3,4-diaminopyridine and intravenous immunoglobulin by his primary physician. However, no improvement could be observed.

Lung cancer was suspected based on the above-mentioned phenomena; thus, the patient was referred to our department. On admission, he could not walk by himself. The laboratory data were as follows: aspartate aminotransferase $21 \mathrm{IU} / \mathrm{l}$, alanine aminotransferase $10 \mathrm{IU} / \mathrm{l}$, blood urea nitrogen 30 $\mathrm{mg} / \mathrm{dl}$, serum creatinine $7.04 \mathrm{mg} / \mathrm{dl}$, neuron-specific enolase $57.5 \mathrm{ng} / \mathrm{ml}$, and pro-gastrin-releasing peptide $1,107.3 \mathrm{pg} / \mathrm{ml}$. We aspirated a subcarinal lymph node and diagnosed SCLC by endobronchial ultrasonography. The disease was classified as stage IIIB (T0N3M0).

CBDCA (300 mg/m² on day 1$)$ and VP-16 (50 mg/m² on days 1 and 3$)$ were administered for 4 cycles, followed by a dose reduction for the further courses. Four-hour HD was carried out $1 \mathrm{~h}$ after giving the regimen [8].

On day 1, the patient's blood pressure became unstable after the administration of the anticancer drugs, with the systolic and diastolic blood pressures measured being 64 and $36 \mathrm{~mm} \mathrm{Hg}$, respectively. As a result, HD was performed for only $1.5 \mathrm{~h}$ with etilefrine. HD could not be performed on day 2 because of continuing hypotension. However, on day 3 , both chemotherapy and HD were administered since his blood pressure completely recovered. There was no new change in the echocardiographic findings between before and after chemotherapy. The ultrafiltrable platinum level was measured by flameless atomic absorption spectrometry. The area under the blood concentrationtime curve of the patient's serum carboplatin level was 15 . The concentration of total serum platinum was measured $1,2.5,24,48$ and $52 \mathrm{~h}$ after chemotherapy administration (fig. 1). He was given platelet transfusion on days 8,10,16 and 23 due to thrombocytopenia (grade 4), and granulocyte colony-stimulating factor was used for febrile neutropenia (grade 4, days 11-25). The patient also developed pneumonia at the time of neutropenia, requiring antibiotics.

His lower extremity muscle weakness dramatically improved to the extent that he was able to kneel down by himself. On day 30, the mediastinal lymph nodes were decreased in size $(53 \%$ shrinkage) on CT. On day 31, both neuron-specific enolase $(4.3 \mathrm{ng} / \mathrm{ml})$ and pro-gastrin-releasing peptide levels $(373.4 \mathrm{pg} / \mathrm{ml})$ were reduced. We assumed that the chemotherapy had induced a partial response. However, the patient could not continue the chemotherapy regimen because his general condition deteriorated due to the severe hematological adverse effects of chemotherapy and persistent hypotension. He died of progressive SCLC on day 94. 


\section{Discussion}

CBDCA has been reported to have a favorable profile for the treatment of HD patients because the platinum level in the plasma of HD patients receiving CBDCA is much more filterable than is the case with cisplatin [9]. The administered dose of 300 $\mathrm{mg} / \mathrm{m}^{2} \mathrm{CBDCA}$ was comparable to the dose generally used in patients with normal renal function and seemed to be appropriate for achieving sufficient efficacy in SCLC patients $[8,10]$. Relatively high-dose chemotherapy using CBDCA and VP-16 for patients with SCLC undergoing HD has been reported [8]. Therefore, we administered a high dose of CBDCA and VP-16 under the informed consent of the patient and the patient's guardian. However, more patients who were treated with a high dose of CBDCA experienced severe adverse effects of chemotherapy than patients on a low dose [8]. Although the regimen was effective in the present patient, he could not undergo scheduled HD and experienced severe hematologic adverse effects that prevented the administration of further chemotherapy.

The patient's hypotension was due to both LEMS and the chemotherapeutic agents. LEMS has previously been reported to induce various symptoms, such as muscle weakness, autonomic dysfunction and cranial nerve symptoms [11]. In addition, it is known that CBDCA and VP-16 can cause hypotension [12-14]. Therefore, we concluded that the combination of the high concentration of chemotherapeutic agents and LEMS caused the severe hypotension that prevented the patient from undergoing HD. In other words, there was a detrimental cycle in which the high concentration of CBDCA led to hypotension, preventing the use of HD, which resulted in an inability to remove the excess platinum, maintaining the high serum concentration for a longer time than normal. Therefore, physicians should be cautious when using chemotherapy in patients presenting with SCLC and combined LEMS because it can lead to hypotension, which is exacerbated in patients needing HD.

We herein reported the first case of a patient with SCLC and LEMS who was treated with CBDCA and VP-16 while undergoing HD. As a result of the onset of severe hypotension due to the chemotherapy, the patient could not continue HD, which in turn led to further toxicity. More careful consideration should be given when selecting the treatment regimen and dose for patients with SCLC and combined LEMS with CRF. 


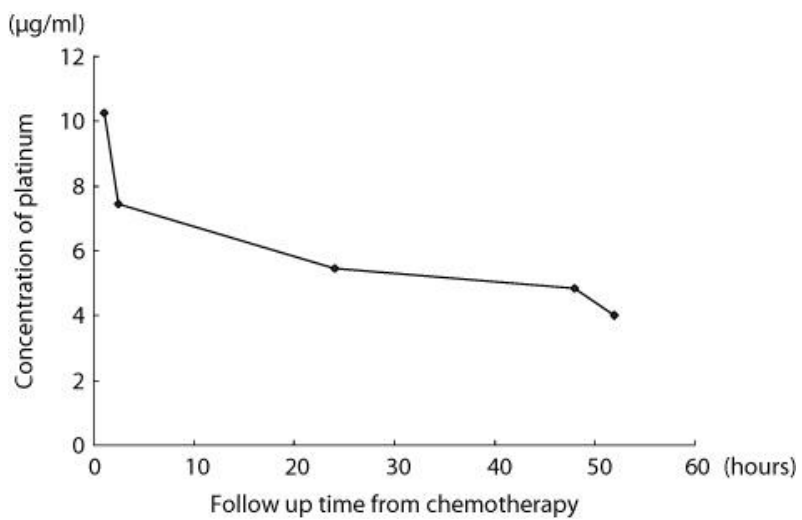

Fig. 1. Platinum concentration in the patient's serum. The concentration after $1 \mathrm{~h}$ following chemotherapy administration was $10.27 \mu \mathrm{g} / \mathrm{ml}$. The patient could not undergo HD; thus, the concentration after $24 \mathrm{~h}$ was extremely high $(5.44 \mu \mathrm{g} / \mathrm{ml})$.

\section{References}

1 Suzuki S: Lambert-Eaton myasthenic syndrome (LEMS). Brain Nerve 2010;62:419-426.

-2 Elrington GM, Murray NMF, Spiro SG, Newsom-Davis J: Neurological paraneoplastic syndromes in patients with small cell lung cancer: a prospective survey of 150 patients. J Neurol Neurosurg Psych 1991;54:764-767.

-3 Tim RW, Massey JM, Sanders DB: Lambert-Eaton myasthenic syndrome: electrodiagnostic findings and response to treatment. Neurology 2000;54:2176-2178.

4 Nakamura S, Kawagishi Y, Kato S, Tsuji H, Takegawa K, Fukuoka J: A long-term survival case of small-cell lung cancer with Lambert-Eaton myasthenic syndrome without anticancer therapy (in Japanese). Nihon Kokyuki Gakkai Zasshi 2010;48:918-922.

5 Sanders DB, Massey JM, Sanders LL, Edwards LJ: A randomized trial of 3,4-diaminopyridine in Lambert Eaton myasthenic syndrome. Neurology 2000;54:603-607.

-6 Donofrio PD, Berger A, Brannagan TH 3rd, Bromberg MB, Howard JF, Latov N, Quick A, Tandan R: Consensus statement: the use of intravenous immunoglobulin in the treatment of neuromuscular conditions report of the AANEM ad hoc committee. Muscle Nerve 2009;40:890-900.

-7 Maisonneuve P, Agodoa L, Gellert R, Stewart JH, Buccianti G, Lowenfels AB, Wolfe RA, Jones E, Disney AP, Briggs D, McCredie M, Boyle P: Cancer in patients on dialysis for end-stage renal disease: an international collaborative study. Lancet 1999;354:93-99.

-8 Inoue A, Saijo Y, Kikuchi T, Gomi K, Suzuki T, Maemondo M, Miki M, Sato T, Nukiwa T: Pharmacokinetic analysis of combination chemotherapy with carboplatin and etoposide in small-cell lung cancer patients undergoing hemodialysis. Ann Oncol 2004;15:51-54.

9 Go RS, Adjei AA: Review of the comparative pharmacology and clinical activity of cisplatin and carboplatin. J Clin Oncol 1999;17:409-422.

10 O’Neill JH, Murrray NM, Newsom-Davis J: The Lambert-Eaton myasthenic syndrome. A review of 50 cases. Brain 1998;111:577-596.

11 Haraguchi N, Satoh H, Ogawa R, Hashimoto Y, Sekizawa K: Chemotherapy in a patient with small cell lung cancer undergoing haemodialysis. Clin Oncol 2005;17:663-668.

12 Markman M, Kennedy A, Webster K, Elson P, Peterson G, Kulp B, Belinson J: Clinical features of hypersensitivity reactions to carboplatin. J Clin Oncol 1999;17:1141.

$\checkmark 13$ Makrilia N, Syrigou E, Kaklamanos I, Manolopoulos L, Salf MW: Hypersensitivity reactions associated with platinum antineoplastic agents: a systematic review. Met Based Drugs 2010;2010:1-10.

14 Gillan MS: Hypersensitivity reactions to chemotherapeutic drug. Clin Rev Allergy Immunol 2010;24:253-262. 